Proyecciones Journal of Mathematics

Vol. 37, No 1, pp. 1-18, March 2018.

Universidad Católica del Norte

Antofagasta - Chile

\title{
The fixed point and the common fixed point properties in finite pseudo-ordered sets
}

\author{
Abdelkader Stouti \\ University Sultan Moulay Slimane, Morocco \\ Received : December 2014. Accepted : November 2017
}

\begin{abstract}
In this paper, we first prove that every finite nonempty pseudoordered with a least element has the least fixed point property and the least common fixed point property for every finite commutative family of self monotone maps. Dually, we establish that a finite nonempty pseudo-ordered with a greatest element has the greatest fixed point property and the greatest common fixed point property for every finite commutative family of self monotone maps. Secondly, we prove that every monotone map $f$ defined on a nonempty finite pseudo-ordered $(X, \unrhd)$ has at least a fixed point if and only if there is at least an element a of $X$ such that the subset of $X$ defined by $\left\{f^{n}(a): n \in \mathbb{N}\right\}$ has a least or a greatest element. Furthermore, we show that the set of all common fixed points of every finite commutative family of monotone maps defined on a finite nonempty complete trellis is also a nonempty complete trellis.
\end{abstract}

2010 Mathematics Subject Classification. 06B23, 54C60, 47H10.

Keywords and phrases : Pseudo-ordered set, trellis, complete trellis, monotone map, fixed point property, least fixed point property, greatest fixed point property, common fixed point property. 


\section{Introduction}

It is well known that partially ordered sets play an important role in various fields (for example see: [9]). In the last decades, several authors studied the fixed point property and the common fixed property for the category of partially ordered sets and self monotone maps (for examples see: [1-6], [11], [13-18] and [22, 23]). In 1971, H. Skala [19, 20] gave two fixed points theorems in the case of pseudo-ordered sets (see [Theorems 36 and 37, 20]). Later on, S. Parameshwara Bhatta and all [7-10] studied the fixed point property in pseudo-ordered sets. Recently in [21], the present author and A. Maaden established some results concerning the existence of the least and the greatest fixed points in the general case of pseudo-ordered sets. In the present paper, without using the existence of maximal element or any other hypothesis of completeness, we study the existence of the least and the greatest fixed point properties, the common fixed property and the least and the greatest common fixed point properties for the category of self monotone maps defined on finite nonempty pseudo-ordered sets. In this way, we first prove that every finite nonempty pseudo-ordered $(X, \unrhd)$ with a least element $\ell$ has the least fixed point property. More precisely, we prove that if $f:(X, \unrhd) \rightarrow(X, \unrhd)$ is a monotone map, then there exists a naturel number $n_{0}$ such that $f^{n_{0}}(\ell)=\min (F i x(f))=\max \left\{f^{n}(\ell): n \in \mathbb{N}\right\}$ (see Theorem 3.1). Then, a finite nonempty pseudo-ordered $(X, \unrhd)$ has the least fixed point property if and only if $(X, \unrhd)$ has a least element. Dually, we establish that if $(X, \unrhd)$ is a finite pseudo-ordered set with a greatest element $g$ and $f:(X, \unrhd) \rightarrow(X, \unrhd)$ is a monotone map, then there exists a naturel number $m_{0}$ such that $f^{m_{0}}(g)=\max (F i x(f))=\min \left\{f^{n}(g): n \in \mathbb{N}\right\}$ (see Theorem 3.5). So, a finite nonempty pseudo-ordered has the greatest fixed point property if and only if it has a greatest element. Consequently, we obtain that every nonempty finite pseudo-ordered with a least or a greatest element has the fixed point property. On the other hand, it is well known that if $(X, \leq)$ is a nonempty finite partially ordered set and $f:(X, \leq) \rightarrow$ $(X, \leq)$ is a monotone map, then $f$ has at least a fixed point if and only if there exists an element $a \in X$ which is comparable with its image $f(a)$ (for example see: [2] and [17]). In this work, we show that the last result is not true in the general case of finite pseudo-ordered sets. However, we prove that if $(X, \unrhd)$ is a finite pseudo-ordered $(X, \unrhd)$ and $f:(X, \unrhd) \rightarrow(X, \unrhd)$ is a monotone map, then $f$ has a fixed point if and only if there is at least an element $a$ of $X$ such that the subset $\left\{f^{n}(a): n \in \mathbb{N}\right\}$ has a least or a greatest element (see Theorem 4.1). For the existence of the least common fixed 
point of a commutative family $\left\{f_{1}, \ldots, f_{n}\right\}$ of monotone maps defined on nonempty finite pseudo-ordered $(X, \unrhd)$ with a least element, $\ell$ we establish that the element $\left(f_{1}^{p_{1}} \circ f_{2}^{p_{2}} \circ \ldots \circ f_{n}^{p_{n}}\right)(\ell)$ is the least common fixed point of the family $\left\{f_{1}, \ldots, f_{n}\right\}$ where $f_{i}^{p_{i}}(\ell)=\min \left(F i x\left(f_{i}\right)\right)$ for every $i=1, \ldots, n$ (see Theorem 5.3). Dually, we prove that if $\left\{f_{1}, \ldots, f_{n}\right\}$ is a finite commutative family of monotone self maps defined on a finite nonempty pseudo-ordered $(X, \unrhd)$ with a greatest element $g$, then the element $\left(f_{1}^{q_{1}} \circ f_{2}^{q_{2}} \circ \ldots \circ f_{n}^{q_{n}}\right)(g)$ is the greatest common fixed point of the family $\left\{f_{1}, \ldots, f_{n}\right\}$ where $f_{i}^{q_{i}}(g)=$ $\max \left(F i x\left(f_{i}\right)\right)$ for every $i=1, \ldots, n$ (see Theorem 5.5). Furthermore, we prove that the set of all common fixed points of every finite commutative family of monotone maps defined on a nonempty complete trellis is also a nonempty complete trellis (see Theorem 5.10).

\section{Preliminaries}

Let $X$ be a nonempty set and $\unrhd$ be a binary relation defined on its. If the binary relation $\unrhd$ is reflexive and antisymmetric, we say that $(X, \unrhd)$ is a pseudo-ordered set or a psoset.

Let $A$ be a nonempty subset of a psoset $(X, \unrhd)$.

An element $u$ of $X$ is said to be an upper bound of $A$ if $x \unrhd u$ for every $x \in A$. An element $s$ of $X$ is called a greatest element or the maximum of $A$ and denoted by $s=\max (A, \unrhd)$ if $s$ is an upper bound of $A$ and $s \in A$.

An element $v$ of $X$ is said to be a lower bound of $A$ if $v \unrhd x$ for every $x \in A$. An element $\ell$ of $X$ is called a least or the minimum element of $A$ and denoted by $\ell=\min (A, \unrhd)$ if $\ell$ is a lower bound of $A$ and $\ell \in A$.

When the least upper bound (l.u.b.) $s$ of $A$ exists, we shall denoted its by $s=\sup (A, \unrhd)$. Dually if the greatest lower bound (g.l.b.) of $A$ exists, we shall denoted its by $\ell=\inf (A, \unrhd)$.

Note that the greatest lower bound and the least upper bound when they exist they are unique.

A psoset $(X, \unrhd)$ is said to be a trellis if every pair of elements of $(X, \unrhd)$ has a greatest lower bound (g.l.b) and a least upper bound (l.u.b). A psoset $(X, \unrhd)$ is said to be a complete trellis if every nonempty subset of $X$ has a g.l.b and a l.u.b. For more details for these notions can be found in H.L. Skala $[19,20]$. 
Let $(X, \unrhd)$ be a nonempty pseudo-ordered sets and let $f: X \rightarrow X$ be a map. We shall say that $f$ is monotone if for every $x, y \in X$, with $x \unrhd y$, then we have $f(x) \unrhd f(y)$.

An element $x$ of $X$ is said to be a fixed point of a map $f: X \rightarrow X$ if $f(x)=x$. The set of all fixed points of $f$ is denoted by Fix $(f)$.

\section{Example.}

Let $A$ the set defined by $A=\{0, a, b, c\}$. We define a pseudo-order relation on $A$ by setting:

(i) for every $x \in A$, we have $0 \unrhd x$ and

(ii) $a \unrhd b \unrhd c \unrhd a$.

Then, $(A, \unrhd)$ is a trellis having the minimum element 0 but $(A, \unrhd)$ is not complete.

Definition 2.1. Let $(X, \unrhd)$ be a nonempty pseudo-ordered set. We say that $(X, \unrhd)$ has the fixed point property if for every monotone map $f$ : $(X, \unrhd) \rightarrow(X, \unrhd)$ the set Fix $(f)$ is nonempty.

Definition 2.2. Let $(X, \unrhd)$ be a nonempty pseudo-ordered set. We say that $(X, \unrhd)$ has the least fixed point property if for every monotone map $f:(X, \unrhd) \rightarrow(X, \unrhd)$ the set Fix $(f)$ is nonempty and has a least element.

Definition 2.3. Let $(X, \unrhd)$ be a nonempty pseudo-ordered set. We say that $(X, \unrhd)$ has the greatest fixed point property if for every monotone map $f:(X, \unrhd) \rightarrow(X, \unrhd)$ the set Fix $(f)$ is nonempty and has a greatest element.

\section{The least and greast fixed point property in finite pseudo- ordered sets}

In this section, we shall study the least and the greatest fixed point properties for finite nonempty pseudo-ordered sets. First, we shall show the following.

Theorem 3.1. Let $(X, \unrhd)$ be a nonempty finite pseudo-ordered set with a least element $\ell$. Then, $(X, \unrhd)$ has the least fixed point property. Moreover for every monotone map $f:(X, \unrhd) \rightarrow(X, \unrhd)$ we have

$$
\min (F i x(f), \unrhd)=\max \left\{f^{n}(\ell): n \in \mathbb{N}\right\}=f^{n_{0}}(\ell)
$$

where

$$
n_{0}=\min \left\{n: n \in \mathbb{N} \text { and } f^{n+1}(\ell)=f^{n}(\ell)\right\} .
$$


Proof. Let $(X, \unrhd)$ be a nonempty pseudo-ordered set with a least element $\ell$ and $f:(X, \unrhd) \rightarrow(X, \unrhd)$ be a monotone map. Let $P$ the following subset of $X$ defined by:

$$
P=\left\{f^{n}(\ell): n \in \mathbb{N}\right\} .
$$

As $P$ is a subset of $X$ and by our hypothesis $X$ is finite, so $P$ is a nonempty finite set. Then, there is $n, p \in \mathbb{N}$ such that $n \neq p$ and $f^{n}(\ell)=f^{p}(\ell)$.

First case. $n<p$.

Claim 1. We have: Fix $(f) \neq \emptyset$. Indeed, as $f$ is a monotone map, then by induction for every $q \in \mathbb{N}$ the map $f^{q}$ is monotone. Since by our hypothesis $\ell$ is the least element of $(X, \unrhd)$ and $n+1 \leq p$, so $\ell \unrhd f^{p-n-1}(\ell)$. Now, as $f^{n+1}$ is a monotone map, then we get $f^{n+1}(\ell) \unrhd f^{p}(\ell)$. On the other hand, by our hypothesis we know tat $f^{n}(\ell)=f^{p}(\ell)$. Hence, we get $f^{n+1}(\ell)=f^{p+1}(\ell)$. So, we obtain $f^{p+1}(\ell) \unrhd f^{p}(\ell)$. Since $\ell \unrhd f(\ell)$ and $f^{p}$ is a monotone map, then $f^{p}(\ell) \unrhd f^{p+1}(\ell)$. Therefore, by antisymmetry of the pseudo-order relation $\unrhd$, we get $f^{p+1}(\ell)=f^{p}(\ell)$. Hence, we have $f\left(f^{p}(\ell)\right)=f^{p}(\ell)$. Thus, $f^{p}(\ell)$ is a fixed point of $f$. So, $F i x(f) \neq \emptyset$.

Claim 2. The set Fix $(f)$ has a least element. Indeed as by Claim 1 we have $f\left(f^{p}(\ell)\right)=f^{p}(\ell)$, then the set $A=\left\{q \in \mathbb{N}: f^{q+1}(\ell)=f^{q}(\ell)\right\}$ is a nonempty subset of $\mathbb{N}$. So $A$ has a least element, $n_{0}$, say. We claim that $f^{n_{0}}(\ell)$ is the least fixed point of $f$. Indeed, if $x \in F i x(f)$, so as $\ell \unrhd x$ and $f^{n_{0}}$ is a monotone map, hence we get $f^{n_{0}}(\ell) \unrhd x$. Thus $f^{n_{0}}(\ell)$ is the least fixed point of $f$.

Claim 3. We have: $f^{n_{0}}(\ell)=\max \left\{f^{n}(\ell): n \in \mathbb{N}\right\}$. Indeed, as $\ell$ is the least element of $X$, then we have $\ell \unrhd f^{n_{0}}(\ell)$. Then as $f^{n}$ is monotone for every $n \in \mathbb{N}$, so we get $f^{n}(\ell) \unrhd f^{n_{0}}(\ell)$. Hence, we deduce that we have

$$
f^{n_{0}}(\ell)=\max \left\{f^{n}(\ell): n \in \mathbb{N}\right\} .
$$

\section{Second case. $p<n$}

Then, by a similar proof in Claim 1 as above, we get $f^{n+1}(\ell)=f^{n}(\ell)$. Therefore the set $\left.A=\left\{q \in \mathbb{N}: f^{q+1}(\ell)\right)=f^{q}(\ell)\right\}$ is a nonempty subset of $\mathbb{N}$. So $A$ has a least element, $m_{0}$, say. Hence, $f^{m_{0}}(\ell)$ is the least fixed point of $f$.

As consequences of Theorem 3.1, we get the following results. 
Corollary 3.2. Let $(X, \leq)$ be a nonempty finite partially ordered set with a least element $\ell$. Then, $(X, \leq)$ has the least fixed point property. Moreover for every monotone map $f:(X, \leq) \rightarrow(X, \leq)$ we have

$$
\min (F i x(f), \leq)=\max \left\{f^{n}(\ell): n \in \mathbb{N}\right\}=f^{n_{0}}(\ell) .
$$

Corollary 3.3. Let $(X, \unrhd)$ be a nonempty finite pseudo-ordered set. Then, $(X, \unrhd)$ has the least fixed point property if and only if $(X, \unrhd)$ has a least element.

Corollary 3.4. Let $(X, \leq)$ be a nonempty finite partially ordered set. Then, $(X, \unrhd)$ has the least fixed point property if and only if $(X, \leq)$ has a least element.

Next, we shall show the existence of the greatest fixed point of self monotone maps defined on nonempty finite pseudo-ordered sets.

Theorem 3.5. Let $(X, \unrhd)$ be a nonempty finite pseudo-ordered set with a greatest element $g$. Then, $(X, \unrhd)$ has the greatest fixed point property. Moreover for every monotone map $f:(X, \unrhd) \rightarrow(X, \unrhd)$ we have

$$
\max (F i x(f), \unrhd)=\min \left\{f^{m}(g): m \in \mathbb{N}\right\}=f^{m_{0}}(g)
$$

where

$$
m_{0}=\min \left\{m: m \in \mathbb{N} \text { and } f^{m+1}(g)=f^{m}(g)\right\} .
$$

Proof. Let $(X, \unrhd)$ be a nonempty pseudo-ordered set with a greatest element $g$ and $f:(X, \unrhd) \rightarrow(X, \unrhd)$ be a monotone map. Let $L$ the following subset of $X$ defined by:

$$
L=\left\{f^{m}(g): m \in \mathbb{N}\right\} .
$$

As $L$ is a subset of $X$ and by our hypothesis $X$ is finite, so $L$ is a nonempty finite set. Then, there is $n, m \in \mathbb{N}$ such that $n \neq m$ and $f^{n}(g)=f^{m}(g)$.

First case. $n<m$.

Claim 1. We have: $F i x(f) \neq \emptyset$. Indeed, as $f$ is a monotone map, then since $g$ is the greatest element of $(X, \unrhd)$ and $n+1 \leq m$, then we get $f^{m-n-1}(g) \unrhd g$. As $f^{n+1}$ is monotone, so we obtain $f^{m}(g) \unrhd f^{n+1}(g)$. On the other hand, we know that $f^{n}(g)=f^{m}(g)$. Hence, we get $f^{n+1}(g)=$ $f^{m+1}(g)$. So, we have $f^{m}(g) \unrhd f^{m+1}(g)$. Since $g$ is the geatest element of $(X, \unrhd)$, so $f(g) \unrhd g$. Then as $f^{m}$ is monotone, we obtain $f^{m+1}(g) \unrhd$ 
$f^{m}(g)$. Therefore, by antisymmetry of the pseudo-order relation $\unrhd$, we get $f^{m+1}(g)=f^{m}(g)$. So, $f\left(f^{m}(g)\right)=f^{m}(g)$. Thus, $f^{m}(g)$ is a fixed point of $f$. Then, Fix $(f) \neq \emptyset$.

Claim 2. The set Fix $(f)$ has a greatest element. Indeed as by Claim 1 we have $f\left(f^{m}(g)\right)=f^{m}(g)$, then the set $B=\left\{q \in \mathbb{N}: f^{q+1}(g)=f^{q}(g)\right\}$ is a nonempty subset of $\mathbb{N}$. So $B$ has a least element, $m_{0}$, say. We claim that $f^{m_{0}}(g)$ is the greatest fixed point of $f$. Indeed, if $x \in F i x(f)$, so as $x \unrhd g$ and $f^{m_{0}}$ is a monotone map, hence we get $x \unrhd f^{m_{0}}(g)$. Thus $f^{m_{0}}(g)$ is the greatest fixed point of $f$.

Claim 3. We have : $f^{m_{0}}(g)=\min \left\{f^{n}(g): n \in \mathbb{N}\right\}$. Indeed, as $g$ is the greatest element of $X$, so we get $f^{m_{0}}(g) \unrhd g$. Then, as $f^{n}$ is monotone for every $n \in \mathbb{N}$, so we obatin $f^{m_{0}}(g) \unrhd f^{n}(g)$. Thus, we have

$$
f^{m_{0}}(g)=\min \left\{f^{n}(g): n \in \mathbb{N}\right\} .
$$

Second case. $m<n$

Then, by a similar proof in Claim 1 as above, we get $f^{n+1}(g)=f^{n}(g)$. Therefore the set $\left.A=\left\{q \in \mathbb{N}: f^{q+1}(g)\right)=f^{q}(g)\right\}$ is a nonempty subset of $\mathbb{N}$. So $A$ has a least element, $n_{0}$, say. Hence, $f^{n_{0}}(g)$ is the greatest fixed point of $f$.

As consequences of Theorem 3.5, we obtain the following results.

Corollary 3.6. Let $(X, \leq)$ be a nonempty finite partially ordered set with a greatest element $g$. Then, $(X, \leq)$ has the greatest fixed point property. Moreover for every monotone map $f:(X, \leq) \rightarrow(X, \leq)$ we have

$$
\max (F i x(f), \leq)=\min \left\{f^{n}(g): n \in \mathbb{N}\right\}=f^{n_{0}}(g) .
$$

Corollary 3.7. Let $(X, \unrhd)$ be a nonempty finite pseudo-ordered set. Then, $(X, \unrhd)$ has the greatest fixed point property if and only if $(X, \unrhd)$ has a greatest element.

Corollary 3.8. Let $(X, \leq)$ be a nonempty finite partially ordered set. Then, $(X, \unrhd)$ has the greatest fixed point property if and only if $(X, \leq)$ has a greatest element.

As consequences of Theorems 3.1 and 3.5, we obatin the following results. 
Corollary 3.9. Let $(X, \leq)$ be a nonempty finite complete trellis. Then, the set of all fixed points of every monotone map $f:(X, \leq) \rightarrow(X, \leq)$ is nonempty and has a least and a greatest elements.

Corollary 3.10. Let $(X, \leq)$ be a nonempty finite complete lattice. Then, the set of all fixed points of every monotone map $f:(X, \leq) \rightarrow(X, \leq)$ is nonempty and has a least and a greatest elements.

\section{Fixed point property in finite pseudo-ordered sets}

It is well known [17] that if $(X, \leq)$ is a finite partially ordered set, then for every monotone map $f: X \rightarrow X$ for which there exists at least an element $a$ of $X$ which is comparable with its image $f(a)$, then $f$ has at least a fixed point. In this section we shall show that the last result is not true in general case of finite pseudo-ordered sets. However, we shall show that if $(X, \unrhd)$ is a finite pseudo-ordered and $f:(X, \unrhd) \rightarrow(X, \unrhd)$ is a monotone map, then $f$ has a fixed point if and only if there is at least an element $a$ of $X$ such that the subset $\left\{f^{n}(a): n \in \mathbb{N}\right\}$ has a least or a greatest element. More precisely, we shall show the following result.

Theorem 4.1. Let $(X, \unrhd)$ be a nonempty finite pseudo-ordered and let $f:(X, \unrhd) \rightarrow(X, \unrhd)$ be a monotone map. Assume that there exists an element $a$ of $X$ such that the subset $\left\{f^{n}(a): n \in \mathbb{N}\right\}$ has a least or a greatest element. Then, Fix $(f) \neq \emptyset$.

Proof. Let $(X, \unrhd)$ be a nonempty pseudo-ordered set and let $f:(X, \unrhd) \rightarrow$ $(X, \unrhd)$ be a monotone map. Assume that there is an elment $a$ of $X$ such that the subset $\left\{f^{n}(a): n \in \mathbb{N}\right\}$ has a least or a greatest element. Let $f:(X, \unrhd) \rightarrow(X, \unrhd)$ be a monotone map.

\section{First case.}

The subset $\left\{f^{n}(a): n \in \mathbb{N}\right\}$ has a least element. Indeed, let $A$ be the following subset of $X$ defined by :

$$
A=\left\{f^{n}(a): n \in \mathbb{N}\right\} .
$$

Then $(A, \unrhd)$ is a finite pseudo-ordered set with a least element. On

the other hand we have $f(A) \subset A$. Since $f$ is monotone on $X$, so $f_{/ A}$ is monotone on $A$. Then, from Theorem $3.1, f_{/ A}$ has a least a fixed point. Then, Fix $(f) \neq \emptyset$. Conversely, if there is $a \in X$ such that $f(a)=a$, then $A=\left\{f^{n}(a): n \in \mathbb{N}\right\}=\{a\}$. 
Second case. The subset $\left\{f^{n}(a): n \in \mathbb{N}\right\}$ has a greatest element. Indeed, let $B$ be the following subset of $X$ defined by :

$$
B=\left\{f^{n}(a): n \in \mathbb{N}\right\} .
$$

Then $(B, \unrhd)$ is a finite pseudo-ordered set with a greatest element. On the other hand we have $f(B) \subset B$. As $f$ is monotone on $X$, so $f_{/_{B}}$ is monotone on $B$. Then, from Theorem $3.5, f_{/_{B}}$ has a greatest fixed point. Thus, we get Fix $(f) \neq \emptyset$. Conversely, if there is $a \in X a$ such that $f(a)=a$, then $A=\left\{f^{n}(a): n \in \mathbb{N}\right\}=\{a\}$.

As a consequence of Theorem 4.1, we get the following.

Corollary 4.2. Let $(X, \leq)$ be a nonempty finite partially ordered set and let $f:(X, \leq) \rightarrow(X, \leq)$ be a monotone map. Assume that there exists at least an element $a$ of $X$ such that $a \leq f(a)$ or $f(a) \leq a$. Then, Fix $(f) \neq \emptyset$.

Next, we shall give a contrexample for the Corolloray 4.2 in the setting of finite pseudo-ordered sets.

\section{Counterexample.}

Let $A$ the set defined by $A=\{a, b, c\}$. We define a pseudo-order relation on $A$ by setting:

(i) for every $x \in A$, we have $x \unrhd x$ and

(ii) $a \unrhd b \unrhd c \unrhd a$.

Then, $(A, \unrhd)$ is a nonempty pseudo-ordered set without any maximal and minimal elements. Now, we define the following map $f: A \rightarrow A$ by setting: $f(a)=b, f(b)=c$ and $f(c)=a$. Note that for every $x \in A$ we have $x \unrhd f(x)$ and the map $f:(A, \unrhd) \rightarrow(A, \unrhd)$ is monotone without any fixed point. That is a counterexample for the case of monotone maps defined on nonempty finite ordered sets.

\section{Common fixed point property in finite pseudo-ordered sets}

In this section we shall study the existence of the least and the geatest common fixed points of finite commutative family of monotone maps defined on a nonempty finite pseudo-ordered set. Furthermore, we prove that the set of all common fixed points of every finite commutative family of monotone maps defined on a nonempty complete trellis is also a nonempty complete trellis.

In this section, we shall need the following definition. 
Definition 5.1. Let $(X, \unrhd)$ be a nonempty pseudo-ordered set and let $\mathcal{F}$ be a family of monotone maps $f$ from $X$ to $X$. We say that $\mathcal{F}$ is a commutative family if for every $f, g \in \mathcal{F}$, we have $f \circ g=g \circ f$.

In this section, we shall need the following lemma whose proof it easy to see.

Lemma 5.2. Let $(X, \unrhd)$ be a nonempty finite pseudo-ordered set and $\mathcal{F}$ be a commutative family of maps $f$ from $X$ to $X$. Then, for every $f, g \in \mathcal{F}$ we have the following:

(i) for every $n \in \mathbb{N}$, we have $f^{n} \circ g=g \circ f^{n}$,

(ii) for every $n, m \in \mathbb{N}$, we have $f^{n} \circ g^{m}=g^{m} \circ f^{n}$.

(iii) for every $n \in \mathbb{N}$, we have $F i x(f) \subset F i x\left(f^{n}\right)$.

(iv) if $g=f_{1}^{p_{1}} \circ f_{2}^{p_{2}} \circ \ldots \circ f_{n}^{p_{n}}$ with $p_{i} \in \mathbb{N}, f_{i} \in \mathcal{F}$ is monotone for $i=1,2, \ldots, n$, and $n \in \mathbb{N}$, then $g$ is a monotone map.

(v) if $g=f_{1}^{p_{1}} \circ f_{2}^{p_{2}} \circ \ldots \circ f_{n}^{p_{n}}$ with $p_{i} \in \mathbb{N}, f_{i} \in \mathcal{F}$ for $i=1,2, \ldots, n$, then $g(F i x(f)) \subset$ Fix $(f)$ for any $f \in \mathcal{F}$.

By using Theorem 3.1, we get the following result.

Theorem 5.3. Let $(X, \unrhd)$ be a nonempty finite pseudo-ordered set with a least element, $\ell$ and let $\left\{f_{1}, \ldots, f_{n}\right\}$ be a finite commutative family of monotone maps defined on $X$. Then, the set of all common fixed points of the family $\left\{f_{1}, \ldots, f_{n}\right\}$ is nonempty and has a least element. Moreover, we have

$$
\min \left(F i x\left(\left\{f_{1}, \ldots, f_{n}\right\}\right)=\left(f_{1}^{p_{1}} \circ f_{2}^{p_{2}} \circ \ldots \circ f_{n}^{p_{n}}\right)(\ell),\right.
$$

where $f_{i}^{p_{i}}(\ell)=\min \left(F i x\left(f_{i}\right)\right)$ for every $i=1, \ldots, n$.

Proof. Let $(X, \unrhd)$ be a nonempty finite pseudo-ordered set with a least element, $\ell$ and let $\left\{f_{1}, \ldots, f_{n}\right\}$ be a finite commutative family of monotone maps defined on $X$. From Theorem 3.1, for each $i \in\{1, \ldots, n\}$ there exists $p_{i} \in \mathbb{N}$ such that $f_{i}^{p_{i}}(\ell)=\min \left(F i x\left(f_{i}\right)\right)$. Set $m=\left(f_{1}^{p_{1}} \circ f_{2}^{p_{2}} \circ \ldots \circ f_{n}^{p_{n}}\right)(\ell)$.

So, we have

$$
\begin{gathered}
f_{1}(m)=f_{1}\left(\left(f_{1}^{p_{1}} \circ f_{2}^{p_{2}} \circ f_{3}^{p_{3}} \circ \ldots \circ f_{n}^{p_{n}}\right)(\ell)\right)=\left(f_{1}^{p_{1}+1} \circ f_{2}^{p_{2}} \circ f_{3}^{p_{3}} \ldots \circ f_{n}^{p_{n}}\right)(\ell) \\
=\left(f_{2}^{p_{2}} \circ f_{3}^{p_{3}} \ldots \circ f_{n}^{p_{n}}\right)\left(f_{1}^{p_{1}+1}(\ell)\right) .
\end{gathered}
$$

Since $\left.f_{1}^{p_{1}+1}(\ell)=f\left(f_{1}^{p_{1}}(\ell)\right)\right)=f_{1}^{p_{1}}(\ell)$, then we obtain

$$
f_{1}(m)=\left(f_{1}^{p_{1}} \circ f_{2}^{p_{2}} \circ f_{3}^{p_{3}} \circ \ldots \circ f_{n}^{p_{n}}\right)(\ell)=m .
$$


Now, let $i \in\{2, \ldots, n\}$, so we have

$$
\begin{gathered}
f_{i}(m)=f_{i}\left(\left(f_{1}^{p_{1}} \circ f_{2}^{p_{2}} \circ \ldots \circ f_{n}^{p_{n}}\right)(\ell)\right)=f_{i}\left(\left(f_{1}^{p_{1}} \circ f_{2}^{p_{2}} \circ f_{i-1}^{p_{i-1}} \circ f_{i+1}^{p_{i+1}} \ldots \circ f_{n}^{p_{n}}\left(f_{i}^{p_{i}}(\ell)\right)\right)\right. \\
=\left(f_{1}^{p_{1}} \circ f_{2}^{p_{2}} \circ f_{i-1}^{p_{i-1}} \circ f_{i+1}^{p_{i+1}} \ldots \circ f_{n}^{p_{n}}\right)\left(f_{i}^{p_{i}+1}(\ell)\right) \\
=\left(f_{1}^{p_{1}} \circ f_{2}^{p_{2}} \circ f_{i-1}^{p_{i-1}} \circ f_{i+1}^{p_{i+1}} \ldots \circ f_{n}^{p_{n}}\right)\left(f_{i}^{p_{i}}(\ell)\right)=\left(f_{1}^{p_{1}} \circ f_{2}^{p_{2}} \circ \ldots \circ f_{n}^{p_{n}}\right)(\ell)=m . \\
\text { Thus, } m \in F i x\left(f_{i}\right) \text { for every } i \in\{1, \ldots, n\} . \text { Hence, we get } m \in \bigcap_{i=1}^{i=n} F i x\left(f_{i}\right) .
\end{gathered}
$$

On the other hand, we know that $F i x\left(\left\{f_{1}, \ldots, f_{n}\right\}\right)=\bigcap_{i=1}^{i=n} F i x\left(f_{i}\right)$. Therefore, we obtain $m \in F i x\left(\left\{f_{1}, \ldots, f_{n}\right\}\right)$.

Next, we shall prove that $m=\min F i x\left(\left\{f_{1}, \ldots, f_{n}\right\}\right)$. Let $x \in F i x\left(\left\{f_{1}, \ldots, f_{n}\right\}\right)$. As $\ell$ is the least element of $X$ and the map $f_{1}^{p_{1}}$ is monotone, so, we get $f_{1}^{p_{1}}(\ell) \unrhd x$ for every $x \in F i x\left(\left\{f_{1}, \ldots, f_{n}\right\}\right)$. By Lemma 5.2 we know that the $\operatorname{map} f_{2}^{p_{2}} \circ f_{3}^{p_{3}} \circ \ldots \circ f_{n}^{p_{n}}$ is monotone, so

$$
\left(f_{2}^{p_{2}} \circ f_{3}^{p_{3}} \circ \ldots \circ f_{n}^{p_{n}}\right)\left(f_{1}^{p_{1}}(\ell)\right) \unrhd\left(f_{2}^{p_{2}} \circ f_{3}^{p_{3}} \circ \ldots \circ f_{n}^{p_{n}}\right)(x) .
$$

Thus, we get $m \unrhd x$ for every $x \in F i x\left(\left\{f_{1}, \ldots, f_{n}\right\}\right)$. Therefore, we obtain $m=\min F i x\left(\left\{f_{1}, \ldots, f_{n}\right\}\right)$.

As a consequence of Theorem 5.3, we get the following result.

Corollary 5.4. Let $(X, \leq)$ be a nonempty finite partially ordered set with a least element, $\ell$ and let $\left\{f_{1}, \ldots, f_{n}\right\}$ be a commutative family of monotone maps defined on $X$. Then, the set of all common fixed points of the family $\left\{f_{1}, \ldots, f_{n}\right\}$ is nonempty and has a least element. Moreover, we have

$$
\min \left(F i x\left(\left\{f_{1}, \ldots, f_{n}\right\}\right)=\left(f_{1}^{p_{1}} \circ f_{2}^{p_{2}} \circ \ldots \circ f_{n}^{p_{n}}\right)(\ell),\right.
$$

where $f_{i}^{p_{i}}(\ell)=\min \left(F i x\left(f_{i}\right)\right)$ for every $i=1, \ldots, n$.

Next, by using Theorem 3.5 we obtain the following result.

Theorem 5.5. Let $(X, \unrhd)$ be a nonempty finite pseudo-ordered set with a greatest element, $g$ and let $\left\{f_{1}, \ldots, f_{n}\right\}$ be a finite commutative family of monotone maps defined on $X$. Then, the set of all common fixed points of the family $\left\{f_{1}, \ldots, f_{n}\right\}$ is nonempty and has a greatest element. Moreover, we have

$$
\max \left(\operatorname{Fix}\left(\left\{f_{1}, \ldots, f_{n}\right\}\right)=\left(f_{1}^{q_{1}} \circ f_{2}^{q_{2}} \circ \ldots \circ f_{n}^{q_{n}}\right)(g),\right.
$$

where $f_{i}^{q_{i}}(g)=\max \left(F i x\left(f_{i}\right)\right)$ for every $i=1, \ldots, n$. 
Proof. Let $(X, \unrhd)$ be a nonempty finite pseudo-ordered set with a greatest element, $g$ and let $\left\{f_{1}, \ldots, f_{n}\right\}$ be a finite commutative family of monotone maps defined on $X$. From Theorem 3.5, for each $i \in\{1, \ldots, n\}$ there exists $q_{i} \in \mathbb{N}$ such that $f_{i}^{q_{i}}(g)=\max \left(F i x\left(f_{i}\right)\right)$. Set $m=\left(f_{1}^{q_{1}} \circ f_{2}^{q_{2}} \circ \ldots \circ f_{n}^{q_{n}}\right)(g)$. So, we have

$$
\begin{gathered}
f_{1}(m)=f_{1}\left(\left(f_{1}^{q_{1}} \circ f_{2}^{q_{2}} \circ f_{3}^{q_{3}} \circ \ldots \circ f_{n}^{q_{n}}\right)(g)\right)=\left(f_{1}^{q_{1}+1} \circ f_{2}^{q_{2}} \circ f_{3}^{q_{3}} \ldots \circ f_{n}^{q_{n}}\right)(g) \\
=\left(f_{2}^{q_{2}} \circ f_{3}^{q_{3}} \ldots \circ f_{n}^{q_{n}}\right)\left(f_{1}^{q_{1}+1}(g)\right) .
\end{gathered}
$$

Since $\left.f_{1}^{q_{1}+1}(g)=f\left(f_{1}^{q_{1}}(g)\right)\right)=f_{1}^{q_{1}}(g)$, then we obtain

$$
f_{1}(m)=\left(f_{1}^{q_{1}} \circ f_{2}^{q_{2}} \circ f_{3}^{q_{3}} \circ \ldots \circ f_{n}^{q_{n}}\right)(g)=m .
$$

Now, let $i \in\{2, \ldots, n\}$, so we have

$$
\begin{gathered}
\left.f_{i}(m)=f_{i}\left(f_{1}^{q_{1}} \circ f_{2}^{q_{2}} \circ \ldots \circ f_{n}^{q_{n}}\right)(g)\right)=f_{i}\left(\left(f_{1}^{q_{1}} \circ f_{2}^{q_{2}} \circ f_{i-1}^{q_{i-1}} \circ f_{i+1}^{q_{i+1}} \ldots \circ f_{n}^{q_{n}}\left(f_{i}^{q_{i}}(g)\right)\right)\right. \\
=\left(f_{1}^{q_{1}} \circ f_{2}^{q_{2}} \circ f_{i-1}^{q_{i-1}} \circ f_{i+1}^{q_{i+1}} \ldots \circ f_{n}^{q_{n}}\right)\left(f_{i}^{q_{i}+1}(g)\right) \\
=\left(f_{1}^{q_{1}} \circ f_{2}^{q_{2}} \circ f_{i-1}^{q_{i-1}} \circ f_{i+1}^{q_{i+1}} \ldots \circ f_{n}^{q_{n}}\right)\left(f_{i}^{q_{i}}(g)\right) \\
=\left(f_{1}^{q_{1}} \circ f_{2}^{q_{2}} \circ \ldots \circ f_{n}^{q_{n}}\right)(g)=m .
\end{gathered}
$$

Thus, $m \in F i x\left(f_{i}\right)$ for every $i \in\{1, \ldots, n\}$. Hence, we get $m \in \bigcap_{\substack{i=1 \\ i=n}}^{i=n} F i x\left(f_{i}\right)$.

On the other hand, we know that we have $\operatorname{Fix}\left(\left\{f_{1}, \ldots, f_{n}\right\}\right)=\bigcap_{i=1}^{i=n} \operatorname{Fix}\left(f_{i}\right)$.

Therefore, we obtain $m \in F i x\left(\left\{f_{1}, \ldots, f_{n}\right\}\right)$.

Next, we shall show that $m=\max F i x\left(\left\{f_{1}, \ldots, f_{n}\right\}\right)$. Let $x \in \operatorname{Fix}\left(\left\{f_{1}, \ldots, f_{n}\right\}\right)$. As $g$ is the greatest element of $X$ and by Lemma 5.2 we know that the map $f_{1}^{p_{1}}$ is monotone, so we get $x \unrhd f_{1}^{q_{1}}(g)$. From Lemma 5.2 the map $f_{2}^{p_{2}} \circ f_{3}^{p_{3}} \circ \ldots \circ f_{n}^{p_{n}}$ is monotone, then we obtain

$$
\left(f_{2}^{q_{2}} \circ f_{3}^{q_{3}} \circ \ldots \circ f_{n}^{q_{n}}\right)(x) \unrhd\left(f_{2}^{q_{2}} \circ f_{3}^{q_{3}} \circ \ldots \circ f_{n}^{q_{n}}\right)\left(f_{1}^{q_{1}}(g)\right) .
$$

Thus, we get $x \unrhd m$ for every $x \in F i x\left(\left\{f_{1}, \ldots, f_{n}\right\}\right)$. Therefore, we deduce that we have $m=\max F i x\left(\left\{f_{1}, \ldots, f_{n}\right\}\right)$.

As a consequence of Theorem 5.5, we get the following. 
Corollary 5.6. Let $(X, \leq)$ be a nonempty finite partially ordered set with a greatest element, $g$ and let $\left\{f_{1}, \ldots, f_{n}\right\}$ be a finite commutative family of monotone maps defined on $X$. Then, the set of all common fixed points of the family $\left\{f_{1}, \ldots, f_{n}\right\}$ is nonempty and has a greatest element. Moreover, we have

$$
\max \left(\operatorname{Fix}\left(\left\{f_{1}, \ldots, f_{n}\right\}\right)=\left(f_{1}^{q_{1}} \circ f_{2}^{q_{2}} \circ \ldots \circ f_{n}^{q_{n}}\right)(g),\right.
$$

where $f_{i}^{q_{i}}(g)=\max \left(F i x\left(f_{i}\right)\right)$ for every $i=1, \ldots, n$.

From Theorems 5.3 and 5.5, we get the following result.

Theorem 5.7. Let $(X, \unrhd)$ be a nonempty finite pseudo-ordered set and let $\left\{f_{1}, \ldots, f_{n}\right\}$ be a finite commutative family of monotone maps defined on $X$. Then, the family $\left\{f_{1}, \ldots, f_{n}\right\}$ has at least a common fixed point if and only if there exists at least an element $a \in X$ such that the subset

$$
\left\{\left(f_{1}^{p_{1}} \circ f_{2}^{p_{2}} \circ \ldots \circ f_{n}^{p_{n}}\right)(a): p_{i} \in \mathbb{N} \text { for } i=1, \ldots, n\right\}
$$

has a least or a greatest element.

Proof. Let $(X, \unrhd)$ be a nonempty finite partially ordered set and let $\left\{f_{1}, \ldots, f_{n}\right\}$ be a finite commutative family of monotone maps defined on $X$. Let $A$ be the following subset of $X$ defined by:

$$
A=\left\{\left(f_{1}^{p_{1}} \circ f_{2}^{p_{2}} \circ \ldots \circ f_{n}^{p_{n}}\right)(a): p_{i} \in \mathbb{N} \text { for } i=1, \ldots, n\right\} .
$$

By our hypothesis, we know that $A$ a least or a greatest element.

Claim 1. We have: $f_{i}(A) \subset A$ for every $i=1, \ldots, n$. Indeed, if $p_{i} \in \mathbb{N}$ for $i=1, \ldots, n$, then

$$
f_{1}\left(f_{1}^{p_{1}} \circ f_{2}^{p_{2}} \circ \ldots \circ f_{n}^{p_{n}}\right)(a)=\left(f_{1}^{p_{1}+1} \circ f_{2}^{p_{2}} \circ \ldots \circ f_{n}^{p_{n}}\right)(a) \in A .
$$

Now, let $i \in\{2, \ldots, n\}$, so we have

$$
\begin{gathered}
f_{i}\left(\left(f_{1}^{p_{1}} \circ f_{2}^{p_{2}} \circ \ldots \circ f_{n}^{p_{n}}\right)(a)\right)= \\
f_{i}\left(\left(f_{1}^{p_{1}} \circ f_{2}^{p_{2}} \circ f_{i-1}^{p_{i-1}} \circ f_{i+1}^{p_{i+1}} \ldots \circ f_{n}^{p_{n}}\right)(a)\right)= \\
\left.\left(f_{1}^{p_{1}} \circ f_{2}^{p_{2}} \circ f_{i-1}^{p_{i-1}} \circ f_{i}^{p_{i}+1} \circ f_{i+1}^{p_{i+1}} \ldots \circ f_{n}^{p_{n}}\right)(a)\right) \in A .
\end{gathered}
$$

Hence, $f_{i}(A) \subset A$ for every $i=1, \ldots, n$. 
Claim 2. The family $\left\{f_{1}, \ldots, f_{n}\right\}$ has at least a common fixed point. Indeed, by Claim 1 we know that $f_{i}(A) \subset A$ for every $i=1, \ldots, n$. So, $\left\{f_{1_{/ A}}, \ldots, f_{n_{/ A}}\right\}$ is a commutative family of monotone maps defined on $A$. As by our hypothesis $A$ has a least or a greatest element, then from Theorems 5.3 or 5.5 we deduce that the family $\left\{f_{1_{/ A}}, \ldots, f_{n_{/ A}}\right\}$ has a least or a greatest common fixed point. Therefore, the family $\left\{f_{1}, \ldots, f_{n}\right\}$ has at least a common fixed point.

By using Theorem 5.7, we obtain the following consequence.

Corollary 5.8. Let $(X, \leq)$ be a nonempty finite partially ordered set and let $\left\{f_{1}, \ldots, f_{n}\right\}$ be a finite commutative family of monotone maps defined on $X$. Then, the family $\left\{f_{1}, \ldots, f_{n}\right\}$ has at least a common fixed point if and only if there exists at least an element $a \in X$ such that the subset

$$
\left\{\left(f_{1}^{p_{1}} \circ f_{2}^{p_{2}} \circ \ldots \circ f_{n}^{p_{n}}\right)(a): p_{i} \in \mathbb{N} \text { for } i=1, \ldots, n\right\}
$$

has a least or a greatest element.

Next, without using the existence of maximal element in finite partially ordered sets we obtain the fllowing result.

Corollary 5.9. Let $(X, \leq)$ be a nonempty finite partially ordered set and let $\left\{f_{1}, \ldots, f_{n}\right\}$ be a finite commutative family of monotone maps defined on $X$. Assume that there exists at least an element $a \in X$ such that $\left(a \leq f_{i}(a)\right.$ for every $i=1, \ldots, n)$ or $\left(f_{i}(a) \leq a\right.$ for every $\left.i=1, \ldots, n\right)$. Then, the family $\left\{f_{1}, \ldots, f_{n}\right\}$ has at least a common fixed point.

Proof. Let $(X, \leq)$ be a nonempty finite partially ordered set and let $\left\{f_{1}, \ldots, f_{n}\right\}$ be a finite commutative family of monotone maps defined on $X$. Assume that there exists at least an element $a \in X$ such that $a \leq f_{i}(a)$ for every $i=1, \ldots, n$ or $f_{i}(a) \leq a$ for every $i=1, \ldots, n$. Let $A$ be the following subset of $X$ defined by:

$$
A=\left\{\left(f_{1}^{p_{1}} \circ f_{2}^{p_{2}} \circ \ldots \circ f_{n}^{p_{n}}\right)(a): p_{i} \in \mathbb{N} \text { for } i=1, \ldots, n\right\} .
$$

First case. We have: $a \leq f_{i}(a)$ for every $i=1, \ldots, n$. Then, by induction we get $a \leq f_{1}^{p_{1}}(a)$ for every $p_{1} \in \mathbb{N}$. So, we obtain $f_{2}^{p_{2}}(a) \leq f_{2}^{p_{2}}\left(f_{1}^{p_{1}}(a)\right)$ for every $p_{1}, p_{2} \in \mathbb{N}$. As by our hypothesis we know that $f_{2}^{p_{2}} \circ f_{1}^{p_{1}}=f_{1}^{p_{1}} \circ f_{2}^{p_{2}}$, so we get $f_{2}^{p_{2}}(a) \leq\left(f_{1}^{p_{1}} \circ f_{2}^{p_{2}}\right)(a)$ for every $p_{1}, p_{2} \in \mathbb{N}$. Since $a \leq f_{2}^{p_{2}}(a)$ and from the transivity of the order relation $\leq$, we obtain $a \leq\left(f_{1}^{p_{1}} \circ f_{2}^{p_{2}}\right)(a)$. 
Now assume by induction that we have $a \leq\left(f_{1}^{p_{1}} \circ f_{2}^{p_{2}} \circ \ldots \circ f_{n-1}^{p_{n-1}}\right)(a)$. Then, we get

$$
f_{n}^{p_{n}}(a) \leq f_{n}^{p_{n}}\left(\left(f_{1}^{p_{1}} \circ f_{2}^{p_{2}} \circ \ldots \circ f_{n-1}^{p_{n-1}}\right)(a)\right) .
$$

On the other hand, we know that $f_{n}^{p_{n}} \circ f_{i}^{p_{i}}=f_{i}^{p_{i}} \circ f_{n}^{p_{n}}$ for every $i=1, \ldots, n-$ 1. Hence, we obtain $f_{n}^{p_{n}}(a) \leq\left(f_{1}^{p_{1}} \circ f_{2}^{p_{2}} \circ \ldots \circ f_{n}^{p_{n}}\right)(a)$. As $a \leq f_{n}^{p_{n}}(a)$ and from the transitivity of the order relation $\leq$, we get $a \leq\left(f_{1}^{p_{1}} \circ f_{2}^{p_{2}} \circ \ldots \circ f_{n}^{p_{n}}\right)(a)$. Therefore, we obtain $a=\min (A)$. Then, from Corollary 5.8 the family $\left\{f_{1}, \ldots, f_{n}\right\}$ has at least a common fixed point.

Second case. We have: $f_{i}(a) \leq a$ for every $i=1, \ldots, n$. Then by a similar proof as above we get $\left.\left(f_{1}^{p_{1}} \circ f_{2}^{p_{2}} \circ \ldots \circ f_{n}^{p_{n}}\right)(a)\right) \leq a$ for every $p_{i} \in \mathbb{N}$ and $i=1, \ldots, n$. Thus, we obtain $a=\max (A)$. Then by Corollary 5.8 we deduce the family $\left\{f_{1}, \ldots, f_{n}\right\}$ has at least a common fixed point.

Next, we shall prove that the set of all common fixed points of a finite commutative family $\mathcal{F}$ of monotone maps $f$ defined on a nonempty complete trellis is also a nonempty complete trellis.

Theorem 5.10. Let $(X, \unrhd)$ be a nonempty complete trellis and $\mathcal{F}$ be a finite commutative family of monotone maps $f:(X, \unrhd) \rightarrow(X, \unrhd)$. Then, the set of all common fixed points $\operatorname{Fix}(\mathcal{F})$ of $\mathcal{F}$ is a nonempty complete trellis.

Proof. Let $(X, \unrhd)$ be a nonempty finite complete trellis and let $\mathcal{F}=$ $\left\{f_{1}, \ldots, f_{n}\right\}$ be a finite commutative family of monotone maps $f_{i}:(X, \unrhd) \rightarrow$ $(X, \unrhd)$ for $i=1, \ldots, n$. From Theorem 5.4 , we know that $F i x(\mathcal{F})$ is nonempty and has a least and a greatest element. On the other hand by [Theorem $3.8,10]$, we know that $F i x\left(f_{1}\right)$ is a nonempty complete trellis. Now, we shall show that the set of common fixed points of the family $\left\{f_{1}, f_{2}\right\}$ is a nonempty complete trellis. Indeed, if $x \in F i x\left(f_{1}\right)$, then $f_{2}(x)=$ $f_{2}\left(f_{1}(x)\right)=f_{1}\left(f_{2}(x)\right)$. So, for every $x \in F i x\left(f_{1}\right)$ we have $f_{2}(x) \in F i x\left(f_{1}\right)$. So, $f_{2}\left(F i x\left(f_{1}\right)\right) \subset F i x\left(f_{1}\right)$. Then as Fix $\left(f_{1}\right)$ is a nonempty complete trellis, hence from [Theorem 3.8, 10] the set of all fixed points of $f_{2}$ in Fix $\left(f_{1}\right)$ is a nonempty complete trellis. On the other hand, the set of all fixed points of $f_{2}$ in $\operatorname{Fix}\left(f_{1}\right)$ is equal to $\operatorname{Fix}\left(\left\{f_{1}, f_{2}\right\}\right)$. Thus, the set of common fixed points of the family $\left\{f_{1}, f_{2}\right\}$ is a nonempty complete trellis. By induction, assume that for every $k \in\{2, \ldots, n-1\}$ the set of all common fixed points of the family $\left\{f_{1}, \ldots, f_{k}\right\}$ is a nonempty complete trellis. Let $x \in \operatorname{Fix}\left(\left\{f_{1}, \ldots, f_{n-1}\right\}\right)$. Then, $f_{n}(x)=f_{n}\left(f_{k}(x)\right)=f_{k}\left(f_{n}(x)\right)$ for every $k \in\{1, \ldots, n-1\}$. So, $f_{n}(x) \in F i x\left(f_{k}\right)$ for every $k \in\{1, \ldots, n-1\}$. Thus, 
$f_{n}\left(F i x\left(\left\{f_{1}, \ldots, f_{n-1}\right\}\right)\right) \subset F i x\left(\left\{f_{1}, \ldots, f_{n-1}\right\}\right)$. As by the hypothesis of induction Fix $\left(\left\{f_{1}, \ldots, f_{n-1}\right\}\right)$ is a nonempty complete trellis and $x \unrhd f_{n}(x)$ for every $x \in F i x\left(\left\{f_{1}, \ldots, f_{n-1}\right\}\right)$, then from [Theorem 3.8, 10] we deduce that the set $\operatorname{Fix}\left(\left\{f_{1}, \ldots, f_{n}\right\}\right)=\bigcap_{i=1}^{i=n} F i x\left(f_{i}\right)$ is a nonempty complete trellis.

\section{References}

[1] Abian, A., Fixed point theorems of the mappings of partially ordered sets, Rendiconti del Circolo Mathematico di Palermo 20, pp. 139-142, (1971).

[2] Abian, S. and Brown, A. B., A theorem on partially ordered sets with applications to fixed point theorems, Can. J. Math. 13, pp. 78-82, (1961).

[3] Amann, H., Order structures and fixed points, Ruhr-universitãt, Bochum, mimeographed lecture notes, (1977).

[4] Baclawski, K., A combinatorial proof of a fixed point property, J. Combin. Theory (A) 119, pp. 994-1013, (2012).

[5] Bourbaki, N., Sur le thorme de Zorn, Arch. Math., 2, pp. 434-437, (1940-1950).

[6] Demarr, R., Common fixed points for isotone mappings, Colloq. Math. 13, pp. 45-48, (1964).

[7] Bhatta, S. P. and Shashirekha, H., A characterisation of completeness for Trellises, Algebra univeralis 44, pp. 305-308, (2000).

[8] Bhatta, S. P., Weak chain completeness and fixed point property for pseudo-ordered sets, Czechoslovac Mathematical Journal, 55 (130), pp. 365-369, (2005).

[9] Bhatta, S. P. and Shiju, G., A note on weak chain-completeness and fixed point property for pseudo ordered sets, Advances in discrete mathematics and applications: Mysore, 2008, 119-123, Ramanujan Math. Soc. Lect. Notes Ser. 13, Ramanujan Math. Soc., Mysore, (2010). 
[10] Bhatta, S. P. and Shiju, G., Some fixed point theorems for pseudo ordered sets, Algebra Discrete Math. 11, No. 1, pp. 17-22, (2011).

[11] Brondsted, A., Common fixed points and partial orders, Proc. Amer. Math. Soc. 77, No. 3, pp. 365-368, (1979).

[12] Capard, N., Leclerc, B. and Monjardet B., Finite ordered sets, Concepts, results and uses. Encyclopedia of Mathematics and its Applications, 144. Cambridge University Press, Cambridge, (2012). xii+337 pp.

[13] Knaster, B., Un théorème sur les fonctions d'ensembles, Ann. Soc. Polon. Math. 6 (1928), 133-134.

[14] Lim, T. C., On the largest common fixed point of a commuting family of isotone maps, Discrete and Continuous Dynamical Systems, Series A, Suppl., pp. 621-623, (2005).

[15] Rival, I., A fixed point theorem for finite partially ordered sets. J. Combin. Theory (A) 21(1976), 309-318 (1976).

[16] Schröder, Bernd S. W., Algorithms for the fixed point property, ORDAL '96 (Ottawa, ON), Theoret. Comput. Sci. 217, No. 2, pp. 301-358, (1999).

[17] Schröder, Bernd S. W., Ordered sets. An introduction, Birkhuser Boston, Inc., Boston, MA, (2003). xviii+391 pp.

[18] Schröder, Bernd S. W., The fixed point property for ordered sets. Arab. J. Math. (Springer) 1, No. 4, pp. 529-547, (2012).

[19] Skala, H. L., Trellis theory, Algebra Universalis 1, pp. 218-233, (1971).

[20] Skala, H. L., Trellis theory, Mem. Amer. Math. Soc. 121, Providence, (1972).

[21] Stouti, A. and Maaden, A., Fixed points and common fixed points theorems in pseudo-ordered sets, Proyecciones 32, No. 4, pp. 409-418, (2013).

[22] Tarski, A., A lattice-theoretical fixpoint theorem and its applications, Proc. Amer. Math. Soc. 5, pp. 285-309, (1955).

[23] Wong, James S. W., Common fixed points of commuting monotone mappings, Canad. J. Math., 19, pp. 617-620, (1967). 


\author{
Abdelkader Stouti \\ Center for Doctoral Studies: Sciences and Techniques, \\ Laboratory of Mathematics and Applications, \\ Faculty of Sciences and Techniques, \\ University Sultan Moulay Slimane, \\ P. O. Box 523. Beni-Mellal 23000, \\ Morocco \\ e-mail : stout@fstbm.ac.ma
}

